\title{
A Design of Airbag Controller Test Equipment
}

\author{
JuanJuan $\mathrm{Li}^{1, \mathrm{a}}$, Bo Qu $\mathrm{u}^{1, \mathrm{~b}}$
}

${ }^{1}$ School of Electronic and Information Engineering, Soochow University, Suzhou, 215006, China

a e-mail :lijuanjuan@suda.edu.cn, ${ }^{\text {b }}$ e-mail : qubo@suda.edu.cn

Key Words: airbag controller, GMLAN, GUI

\begin{abstract}
The stability and reliability of airbag controller determines the security of the whole vehicle cabin system, but because of it's complex internal structure, it's hard to test every main function before leaving factory, at the meanwhile, multiple testing may cause damage or unwanted fault codes for products, therefore, the "one-stop" detection equipment is very necessary. This paper introduces a design without manual intervention, switch loads automatically, consistent with the GMLAN communication protocol, and introduces the hardware design, GMLAN protocol and the GUI based on python in detail.
\end{abstract}

\section{Introduction}

With the continuous development of automobile electronic technology, the requirements of automotive intelligent control is becoming more and more higher. As the core component of automotive safety system, airbag controller must have the extremely high reliability and security, this also put forward high request to the test equipment[1]. This paper designs a set of virtual automotive environment, complete a number of testing work on the airbag controller in the end of the production, at the same time with the method of ping-pong operation, the uninterrupted test between products is realized, which greatly improved production efficiency.

\section{System hardware introduction}

The hardware platform of this system is mainly composed of power supply module, microprocessor, load switch circuit, over-current protection, CAN transmitting and receiving circuit and the signal indication circuit. The system hardware diagram are shown in Figure 1.

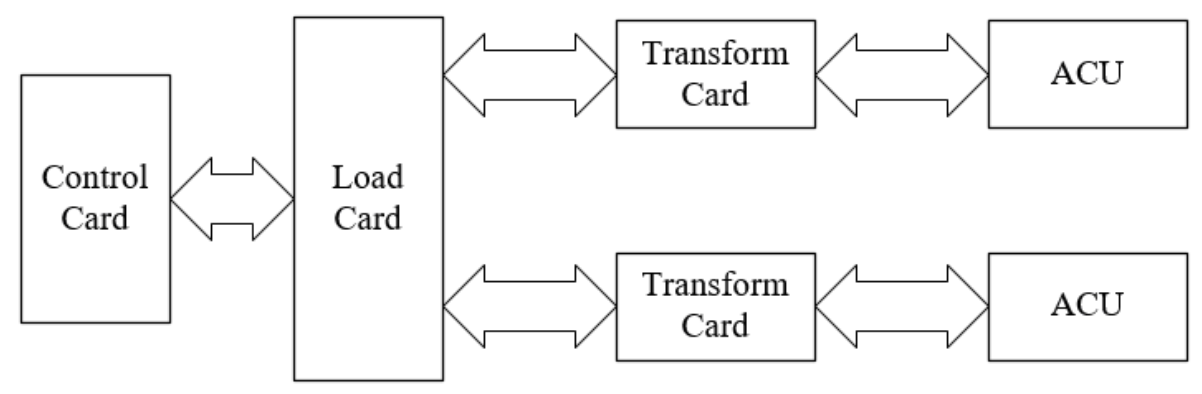

Fig. 1 System hardware diagram

The test platform designed in this paper is based on the 32-bit processor STM32F103RET6 of ST company, its internal resources is very abundant. This design use mainly serial port, CAN controller, GPIO port, etc[2], of which the serial port is used to communicate with GUI, to realize different parameters of products, CAN controller is connected externally with a CAN transceiver MCP2551, to communicate with the airbag controller and read its internal data to judge whether it's abnormal. SPI controller concatenates 4 MBI5025, to realize up to 64 road loading switch. GPIO set the input to test whether the product is in place, and set the output to control the indicator lights[3].

\section{Power supply module}

The scheme the power supply module uses is a primary switching power supply and a after class LDO. The power supply module are shown in Figure 2.The switching power supply uses XL4012, transfer the voltage from $19 \mathrm{~V}$ to $5 \mathrm{~V}$. XL4012 is a $300 \mathrm{KHZ}$ fixed frequency PWM step-down DC - 
DC conversion chip, the output current load capacity can reach $12 \mathrm{~A}$, input voltage range is $5 \sim 32 \mathrm{~V}$, output voltage range is $0.8 \sim 30 \mathrm{~A}$, which can completely satisfy the load requirements of the product. XL4012 enable pin NC foot control the output of chip, when the potential of NC foot is high level (above 1.4V) or dangling, the chip has output, when the potential of NC foot is low level ( under $0.8 \mathrm{~V}$ ), the chip shutoff output. The datum of $\mathrm{FB}$ is $0.8 \mathrm{~V}$, by adjusting the proportion of resistor R5, R7 the output of specified voltage can be realized[4].

The Microprocessor uses 3V3 voltage, so a circuit to transfer from $5 \mathrm{~V}$ to $3 \mathrm{~V} 3$ is needed. This design uses the level conversion chip NCP1117, its internal protection function includes output current limit, ASO compensation and heat shut-off.
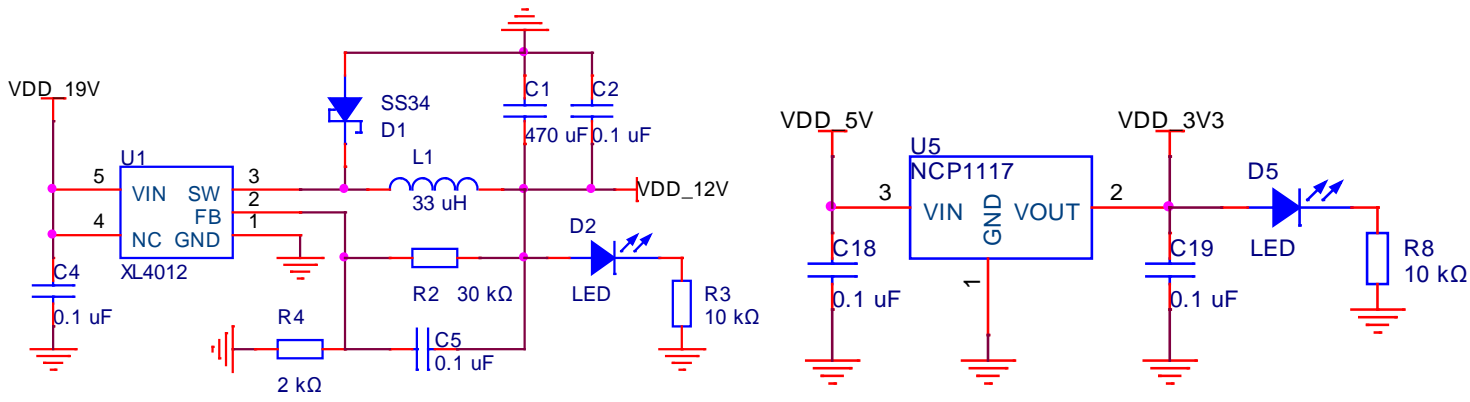

Fig. 2 Power supply module

\section{Load switch circuit and over-current protection circuit}

Airbag controller need to load more, including satellite sensors testing the car's acceleration, 2ohm resistor detecting the capability of airbags explosion, persistence current source simulating whether the safety belt is fine, so it is needed to switch different model according to different load. Normally, the relay is used as the "bridge". The relay has two kinds of drives: parallel and serial. The parallel one uses triode or Darlington tube, the serial one uses the shifting register. The former one is more convenient, but takes more IO of microcontroller, the latter one uses MCU SPI resource and this can achieve the purpose of controlling multiple relays by using three pin. This design uses MBI5025 shifting chip to realize the transfer between parallel and serial.

\section{System software introduction}

\section{GMLAN protocol introduction}

GMLAN is the communication standard of GM, which provides a set of serial bus to realize the mutual communication between the ECU. These bus are based on CAN communication standard, adopting the same data communication policy. The content of the GMLAN recommended scheme includes: using three different communication baud rate on the physical layer or protocol. In view of the high-speed communications, the 500KBPS double CAN bus is adopted. Aiming at medium speed communication, the 95 KBPS double CAN bus is adopted. In view of the low speed communication, the 20 40KBPS single CAN bus is adopted[5].

One message of GMLAN protocol corresponds to one identifier of the CAN, the length of a logical message can be greater than the data volume that is transmitted by physical layer of single CAN. Message can be composed by multiple signal, the length can be from 1 digit to 4095 byte. In this design it's mainly the communication with ECU, divided into single frame transceiver and successive frames transceiver, as shown in Figure 3.

FF、CF、FC、SF means respectively the first frame, successive frame, flow control and single frame, of which FF、CF、FC are normally used together, because the returning data of the multiframe data is normally more than eight, before receiving the rest of the frame, the flow control must be delivered to "notify" ECU to send back the remaining frame data. 


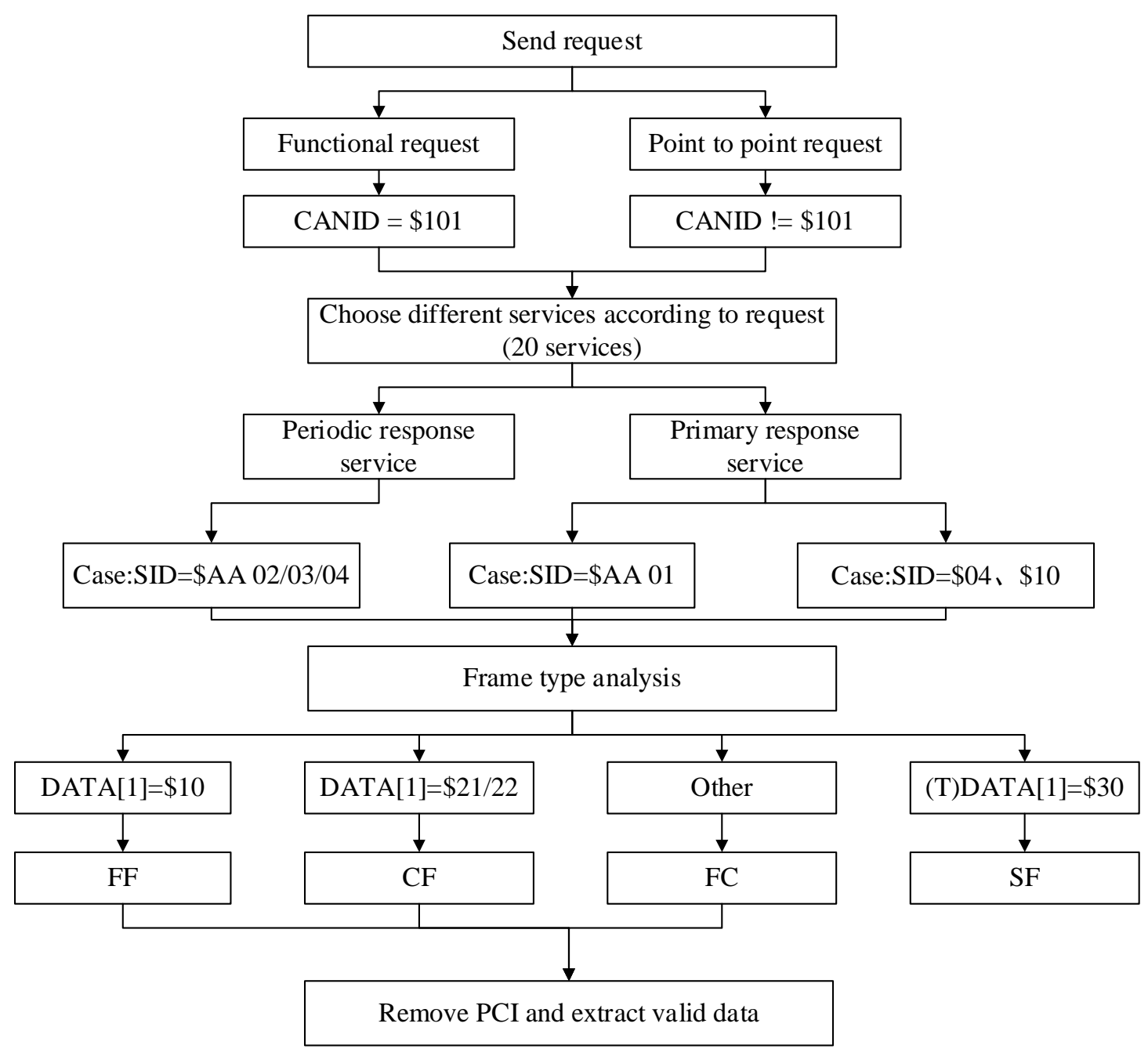

Fig. 3 GMLAN communication process

"Ping-pong" Operation procedure

"Ping-pong” Operation procedure are shown in Figure 4.

A:

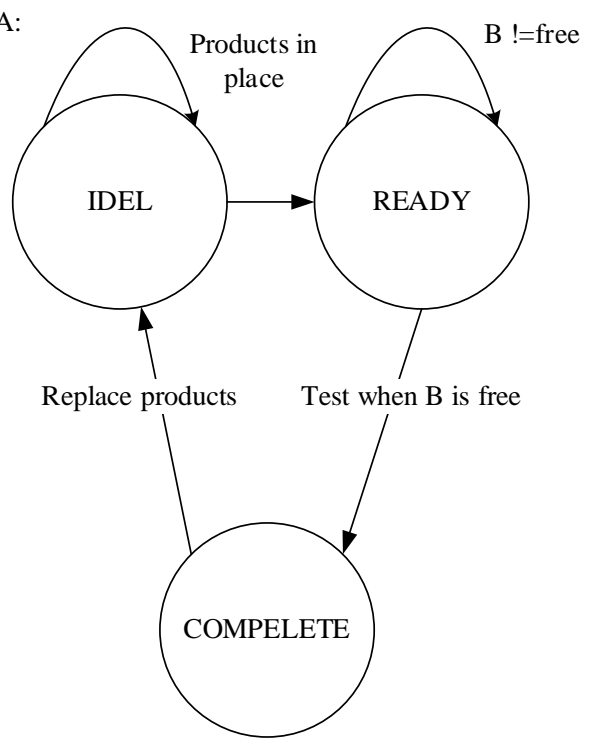

B:

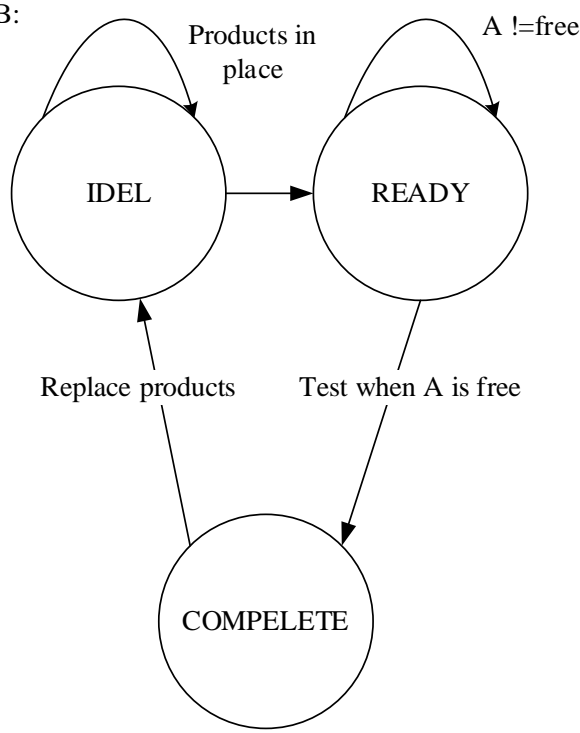

Fig. 4 “Ping-pong” operation procedure 
The specific operation procedure: Fix A product and make it in place, then test A product. In the period of testing, fix B product and make it in place for testing. When A product finish testing and exit the in place state, B product will start testing automatically. In this way, the continuous inspection is implemented for the products under test in A and B products position, which greatly improve the testing efficiency of the factory product. Software need only two state machines to achieve this, which will not be introduced here.

\section{GUI introduction}

A friendly man-machine interface is one of the stand to evaluate the stand or fall of a system. This paper designs a GUI using Python, users can add different configuration files according to different products, transmit configuration files, reveal test results, etc. shown in Figure 5.

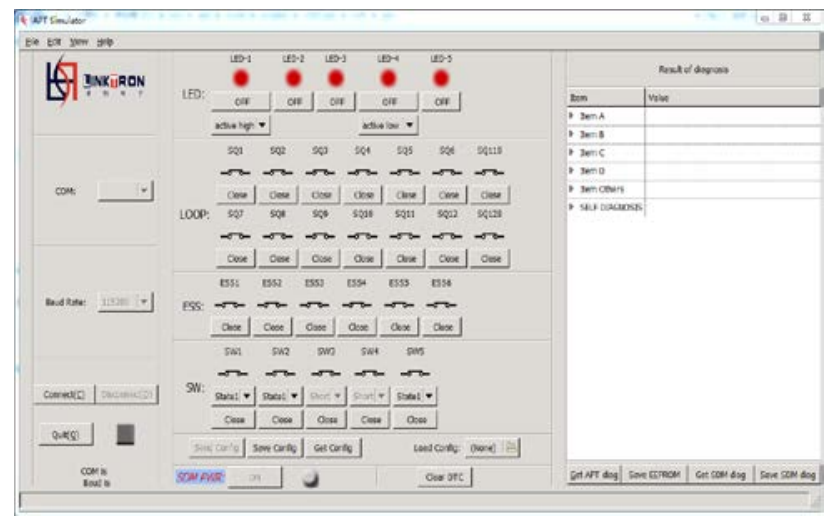

Fig. 5 GUI display

\section{Conclusion}

The airbag controller test equipment designed in this paper has a good stability and practicability in the practical work, it assists effectively the ACU manufacturers in their products inspection and obtains the affirmation from clients. Of course, the development of the test equipment is a process that need gradually improvement, along with the continuous renewal of products, the system is also improved continuously. In the direction of intelligent, we make it to rewrite the configuration file and check the results without using computer or IPC and just a mobile phone or tablet is enough.

\section{References}

[1] Na Chen, Wen He, Automobile safety airbag control core, vol (9), 5-8(2004)

[2] Jinqian Yu, Bin Yu: STM32F series ARM Cortex-M3. Development and application of core micro controller, Beijing Tsinghua University press (2011)

[3] STMicroelectronics. STM32F10xxx reference manual. Doc ID 13902 Rev 11. April, 2010.

[4] XLSEMI Devices. XL4012 Data Sheet. Rev 2.12012.

[5] Xin Liu, Zhiyong Zhang: Multi objective optimization of safety airbag cushioning characteristics in frontal crash. Information system engineering [J], vol (10), 32-34 (2011). 